Journal of Engineering and Applied Sciences 10 (6): 123-136, 2015

ISSN: $1816-949 \mathrm{X}$

(C) Medwell Journals, 2015

\title{
Numerical Simulation of Twin Impinging Jets in Tandem Through a Crossflow
}

\author{
Diana F.C. Vieira, Jorge, M.M. Barata, Fernando M.S.P. Neves and Andre R.R. Silva \\ Universidade Beira Interior, 6200-358 Covilha, Portugal
}

\begin{abstract}
The flow field of ground vortex generated by twin impinging jets in tandem through a crossflow is numerically studied in detail. Numerical simulation and visualization are presented for two turbulent circular jets emerging into a low velocity cross stream, impinging after on a flat surface perpendicular to the geometrical jet nozzle axis. The numerical study is based in experimental studies done early, so all the features of the experimental flow were maintained when the numerical simulation was performed. The Reynolds number used was based on the jet exit conditions of 43.000-105.000, a jet to crossflow velocity ratio of 22.5-43.8 an impinging height of 20.1 jet diameters and an interject spacing's of $S=5 \mathrm{D}$ and $\mathrm{L}=6 \mathrm{D}$. The analysis of the flow was extended to regions and flow conditions for which no measurements have been obtained in last experimental studies, i.e., for velocity ratios of 7.5-60. The numerical results show that for the smallest velocity ratios the jets initially do not mix but remain together in two layers. Three different types of flow regimes were identify, therefore when VSTOL aircrafts operating in ground vicinity, only the regime with strong impingement on ground and with a formation of a ground vortex is relevant. The numerical results allowed to extend the last experimental studies and prove that the deflection of the rear jet is due to the competing influences the wake, the shear layer, the downstream wall jet of the first jet and the crossflow.
\end{abstract}

Key words: Impinging jets, crossflow, tandem, ground vortex, wake

\section{INTRODUCTION}

In external aerodynamics applications such as VSTOL aircrafts, turbulent jets impinging on flat surfaces through a low velocity crossflow are typical of the flow beneath of this type of aircraft. During its landing or near ground hovering phase, the VSTOL aircraft creates a complex three dimensional flow field between the jet streams, the airframe surface and the ground. When ground effect occurs, the lift forces on the aircraft changes cause hot gas re-ingestion into the engine intake and due to the fountain upwash and ground flows, the fuselage skin temperature rises. The unsteadiness of the flow and raise of the temperature cause several problems in the engine performance such as compressor surge or even stall and thrust reduction. In respect to the intake ingestion phenomenon, it is very complex and can be associated with the design and operational parameters such as jet configuration, head wind velocity, jet impingement height or intake configuration. In the case of the hot gas ingestion problem, there are three mechanism involved, i.e., far field ingestion, near field ingestion and ground vortex ingestion. The first mechanism is results of the forward away initially movement of the ground sheet wall jet due to the aircraft movement. This happened because the hot gases after some distance loses its momentum, rising and separating from the ground. The portion of the hot gases that separating from the ground, mixes with the surrounding air and backs again to the intake. The second mechanism, near field ingestion has a much greater impact on hot gas ingestion compared to the first because it directly affected the lift nozzle exits into the surrounding area of the intake being that when exits multiple impinging jets, its impact on the ground plane create a fan shape up wash fountain beneath the aircraft. When the fountain impinges on the underside of the fuselage, flowing from the fuselage to the intake, the engine may sucks the flow to the intake, creating severe temperature distortion to the intake, since, these gases are much hotter than those from the far field ingestion. The latter mechanism is due to the presence of a ground vortex. During a landing or hover the impingement of each downward-directed jet on the ground results in the formation of a wall jet which flows radially from the impinging point along the ground surface. The interaction of this wall jet with the free stream results in the formation of a ground vortex far upstream of the impinging jet. This flow field transports exhaust gases away from the ground and up toward the intake region. The level and intensity of the ingestion resulting from this mechanism depends critically on the forward velocity. If there are two or more adjacent jets, the resulting wall jets meet and a fan-shaped upwash or "fountain" is normally

Corresponding Author: Diana Filipa da Conceicao Vieira, Department of Aerospace Science, University of Beira Interior, 6200-358 Covilha, Portugal 
formed between the jets. The fountain upwash flow depending on its strength and direction affects the forces and moments induced in the aircraft when operating in ground effect. The resulting ground vortex shape is strongly affected and the corresponding induced suckdown effect tends to be reduced by the upload produced by the fountain. In the last 30 or 40 years, this type of complex flow fields have been studied extensively but improve the knowledge are ever required because the aircraft design have been changed since its first design and some problems were solved but others were not. Earlier published work has been concentrated on 1,2 and 3 jets configurations relevant to the AV-8B Harrier II aircraft. In this case when the aircraft operates with small forward movement the configuration of interest are two impinging jets with the direction of the crossflow perpendicular to the line containing their centers.

For the next generation of VSTOL aircrafts F-35 no relevant studies can be found because the impinging jets are aligned with the crossflow and this geometry has not yet been considered. In this case, a vertically oriented lift fan (SDLF) generates a column of cool air that produces the nearly 20,000 pounds of lifting power, along with an equivalent amount of thrust from the vectored rear exhaust (3BSM-Three Bearing Swivel Module). Figure 1 shows the position of the thrust vectoring nozzle and lift fan in the F-35 aircraft. The lift system was successfully demonstrated during a flight test of the X-35B during the Summer of 2001. The complexity of the new VSTOL configuration together with the very stringent requirements has required an enormous amount of $R \& D$ in the last decade. On 12th May 2012, the 200th test flight of the F-35B (BF-3) measurement of stresses on the aircraft during supersonic maneuvers was done. Therefore, most of the published work reported so far has only peripheral relevance to the F35-B/JSF ground effect problem.

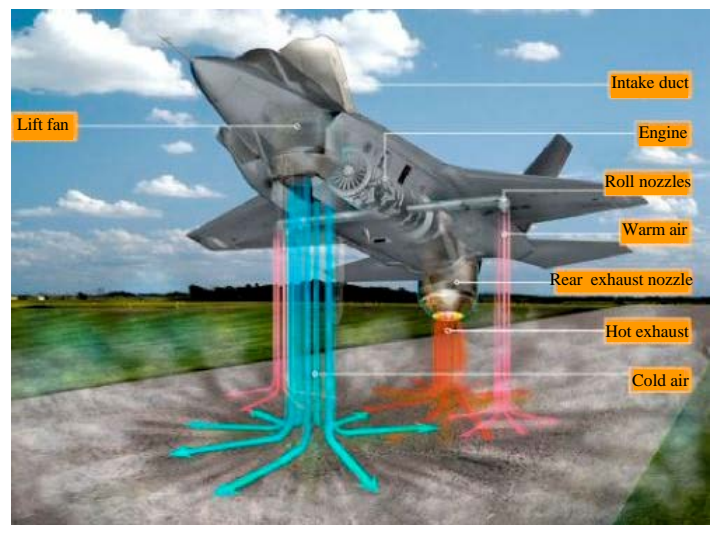

Fig. 1: F-35's thrust vectoring nozzle and lift fan
This study aims presents results that are relevant to the future F-35 VSTOL configuration (Fig. 2), through a detailed analysis of the complex flow field beneath two impinging jets in tandem through a low velocity crossflow.

The present study is a piece of a most comprehensive experimental study on impinging jet flows in ground effect which included two different experimental installations: 2D ground vortex and wind tunnel. Here, the main focus is the extrapolation of the measurements beyond the limits of the latter experimental rig through computational simulations.

Previous detailed measurements of the flow properties for fountain up wash flow are scarce and have been presented essentially in the absence of a crossflow and with the use of probe techniques and more recently with particle image velocimetry. The most relevant works have been reviewed by Barata (1989) and Saripalli (1983) showing high turbulence levels and spreading rates in the fountains (Gilbert, 1983; Nishino et al., 1996). In the case (Nishino et al., 1996), the turbulent normal stress of the axial component made a substantial contribution to the increase in the static pressure on a region near the impingement plate being the turbulence close an axisymmetric state in the stagnation region. Saripalli (1987) reports Laser Doppler Velocity (LDV) measurements including those of shear stress for axisymmetric impinging jets with an distance between the jet axis, $S / D=9$ and 14 with an impinging height, H/D of 3 and 5.5 but again the existence of a crossflow was not considered. Detailed measurements of the velocity characteristics of normal impinging jets on a flat surface (Fig. 3) can be found for single jet configurations for relatively large impingement heights and normally for $\mathrm{H} / \mathrm{D}>10$ using either probe or optical techniques as reviewed for example by Barata et al. (1993). Crabb et al. (1981) reports LDV

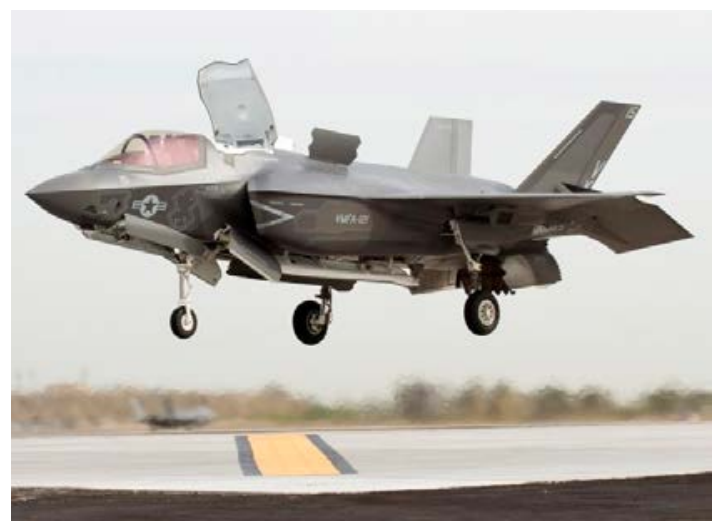

Fig. 2: F-35 VSTOL configuration 


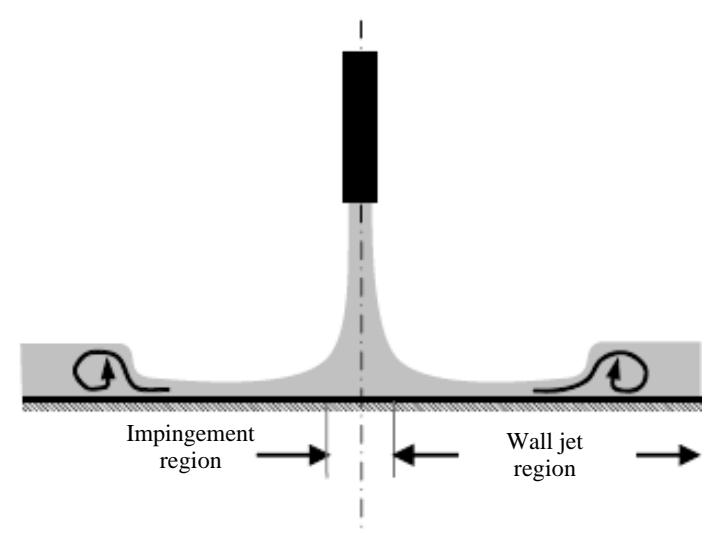

Fig. 3: Schematic of a normal impinging jet on a flat plate or ground (Kate et al., 2007)

measurements including shear stress measurements for values of $H / D=12$ and for velocity ratios up to 2.3. Only Barata (1989) provided detailed LDV measurements for a single jet configuration for a jet with Reynolds number $\operatorname{Re}_{j}=6 \times 10^{4}$, a velocity ratio between the jet, $V_{j}$ and the crossflow, $U_{0}, V_{R}=V_{j} / U_{0}$ of 30,42 and 73 with jet exit above the ground plate 3,4 and 5 diameters. The measurements include time-resolved velocity characteristics along the horizontal, vertical directions with respective correlations in planes parallel to the jet nozzle axis.

Barata (1996a, b) extended their study to multi jet impinging configurations producing upwash fountain flows (Fig. 4) which are the heart of the complex effects produced by VSTOL aircraft when they operate in ground proximity but as far as twin jets are concerned only the geometry with the jets side by side was considered. Experiments on the aerodynamics of jets through a confined crossflow are scarcer and have only been reported for large impingement heights and for low velocity ratios between the jet and the crossflow $\mathrm{V}_{\mathrm{j}} / \mathrm{U}_{0}$. Therefore, these works have only peripheral relevance to the VSTOL ground effect problem with the same geometry of the F-35. Behrouzi and McGuirk (2000) studied the experimental data of the intake in a short take off and vertical landing aircraft in ground effect through laser Doppler measurements. The results confirmed that the flow pattern produced with the impingement height and velocity ratio parameters selected were typical of practically occurring re-ingestion flow fields. Researchers also did this study to obtain results as benchmark validation data for time averaged Turbulence Model based RANS CFD predictions. Kolar et al. (2003) reports a hot-wire anemometry measurements for two different (tandem and side by side) twin jets arrangement for values

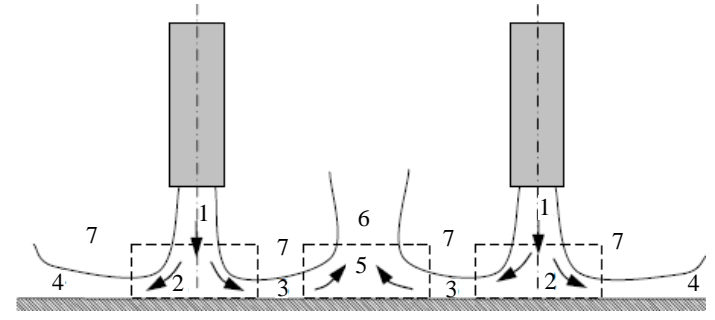

Fig. 4: Schematic of the upwash fountain flow by two impinging jets: 1: Free jet, 2: Jet impingement region, 3: Inner wall jet region, 4: Outer wall jet region, 5: Fountain formation region, 6: Fountain upwash flow and 7: Entrainment (Kate et al., 2007)

of $\mathrm{V}_{\mathrm{j}} / \mathrm{U}_{0}$ to equals 8 . The results show that the vorticity distribution, vortex strength, transverse penetration ability and intensity of vorticity transport were strongly dependent function of the geometrical parameters. More recently a brief survey of the recent studies of twin jets in crossflow was carried out by Kolar and Savory (2007), show that the tadem configurations have rapid mixing and great penetration into the crossflow than side by side configurations.

They also report how the transverse penetration ability and vortex strength are subject to the nozzle arrangement and they found that all cases of twin jets in crossflow lead qualitatively to the some dominant vertical structures, counter rotating vortex pair being responsible by convective entrainment. An important characteristic of two impingement jets with the presence of a crossflow are the fountain upwash flow produced by the collision of the wall jets and the entrainment of ambient air into different regions of the flow (Saripalli, 1983). Kate et al. (2007) studied the spreading flow due to the normal impingement of two closely spaced liquid jets. The visualization results allowed the researchers to conclude that depending on the spacing between the two jets and their relative strength, different kinds of thin film interactions are possible, resulting in a variety of flow pattern. Saddington et al. (2005) also studied the fountain flow for two under expanded, axisymmetric and turbulent impinging jets on a ground plane using Laser Doppler Velocimetry and Particle Image Velocimetry. It was identify the presence of a large scale structures on the fountain flow and at the stagnation region with high degree at asymmetry. Some experimental work published about impingement jet in crossflow not only quantified the flow field but also the heat transfer characteristics. Wae-Hayee et al. (2014) reports oil film technique for values of $\mathrm{V}_{\mathrm{j}} / \mathrm{U}_{0}$ up to 33,5 and 7 for an impinging jeta small jet to plate distance, $H / D=2$. The results show that 
the Nusselt number peak shifts downstream and increases with the increase of crossflow velocity. In order to explore the convective heat transfer of an impinging jet in a crossflow, Li et al. (2014) report a study where the enhancement factor was found to increase with the jet-to-crossflow mass ratio and the Reynolds number but decrease with the jet diameter. The presence of a crossflow was observed to degrade the heat transfer performance. Caliskan and Baskaya (2012) report a laser Doppler anemometry and an infrared thermal imaging study for two different impingement height, $\mathrm{H} / \mathrm{D}=3$ and 12 for a smooth surface and a V-shaped ribs. When the jet to plate spacing was low the V-SR surface showed higher values of turbulent kinetic energy and Nusselt number, being the Nusselt number at the stagnation point decrease with the increase in H/D.

Most of the computational work published on jets with crossflow has been based on integral methods admitting assumptions simplified which are only capable of predicting global effects such as trajectories and jet cross-section shapes, for example Adler and Baron (1979) and Patankar et al. (1979) employed a finite-difference numerical procedure together with a two-equation Turbulence Model to predict a single jet in an unconfined crossflow and obtained good agreement with the experiments by Knowles and Bray (1991) for velocity ratios from 2-10. A similar approach was used by Jones and McGuirk (1980) to calculate the confined flow measured by Kamotani and Greber (1974). The gross features of the flow are well predicted but the calculations appear to exhibit diffusion rates larger than those consistent with measured profiles which can be attributed either to numerical or Turbulence Model errors. Grids up to $20 \times 15 \times 15$ nodes were used but further grid refinement is essential to identify the precise source of disagreement between measurements and predictions. Knowles (1996) has shown that the standard " $k-\varepsilon$ " Model over predicts the spreading rate of a free jet and under predicts the spreading rate of a radial wall jet. Barata et al. (1991) and Worth and Yang (2006) used the steady-RANS or SRANS approach but the results did not capture the turbulent structure of the fountain flow and impingement regions being the predicted turbulent quantities particularly poor. Recently, Yang (2014) reports a numerical study employing the Unsteady-RANS or URANS approach, comparing the results with (Barata et al., 1991). Compared the results with the computational results with the SRANS approach by Worth and Yang (2006), the URANS approach was the one that better agreement with the experimental data. Fan et al. (2006) employed in their numerical studies large eddy simulation and $\mathrm{RNG}$
Turbulence Model. Fan et al. (2006) detected the formation and evolution of the steady or quasi-steady vortical structures by the interaction of an impinging jet in a confined crossflow but without reference to the instantaneous flow patterns by the RNG " $k-\varepsilon$ " Turbulence Model. However, in this two works (Fan et al., 2006) the forming and time evolution characteristics of highly 3D vertical structures are not well understood due to the limitations of the two computational methods being the mixing of the ambient crossflow fluid by the unsteady vertical structures in the near wall regions not fully exposed. Abdel-Fattah (2007) presented a comparison of experimental data and computational simulation about an impinging jet without crossflow for different impingement heights, $3<\mathrm{H} / \mathrm{D}<12$, jet to jet spacing and jet angle, $0^{\circ}<\theta<20^{\circ}$. The results showed that the stagnation primary point moves away in the radial main flow direction with jet angle increase and become stronger with the jet to jet spacing increase. The pressure at the stagnation point located at the middle between the two jets increase with the jet angle but decrease with the Reynolds number. The increase of the impingement height decreases the intensity of the re-circulation zone in the middle of the jets and the jet spreading. An identical numerical study was reported by Yang and Shyu (1998) but the main focus of the study was the study of heat characteristics with the modification of the jet angle. The increase of the jet angle showed a downstream move of the maximum local nusselt number and the maximum pressure. When compared with the Reynolds number, the jet angle proved to have a more significant effect on the recirculation zone. Miao et al. (2009) report a numerical study with different crossflow orientations and jet to surface spacing. The study revealed that the flow exit crossflow e orientation affected significantly the developing jet flow fields and the Nusselt number. Aldabbagh and Sezai (2002a, b) and Aldabbagh and Mohamad (2006) studied the flowfield and the heat transfer for square array impinging jets for different impingement height where the local Nusselt number reduces with the increase of the H/D but the number of jets combined not affected the heat characteristics.

\section{MATERIALS AND METHODS}

Flow configuration: The experiments were performed on a wind tunnel facility designed and constructed for the present work with an exit section of $300 \times 402 \mathrm{~mm}$ that is schematically shown in Fig. 5. The test section was made of Perspex allowing the passage of laser beams. During all the design process special consideration was taken for the boundary layer in which the recommendations by 


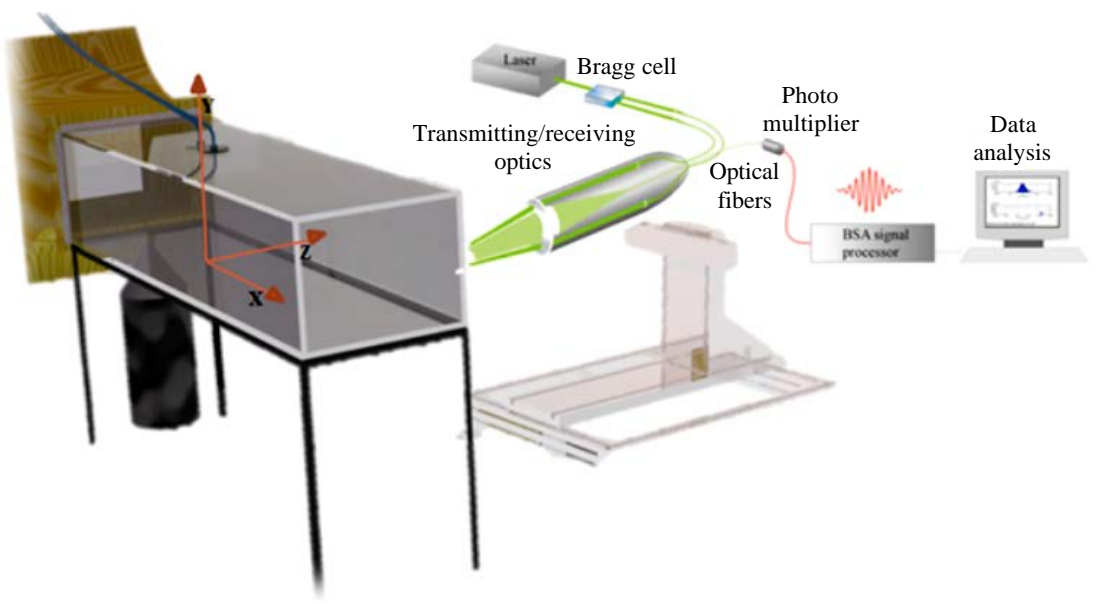

Fig. 5: Experimental set-up

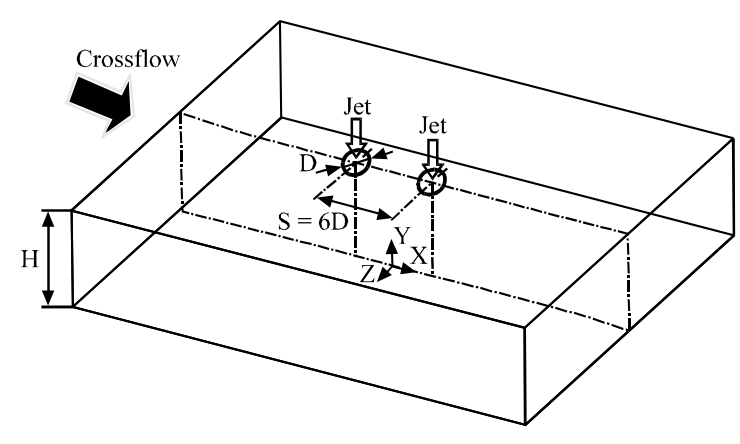

Fig. 6: Geometrical arrangement of the jets

Durst et al. (1976) for open circuit wind tunnels was followed. A fan with $15 \mathrm{~kW}$ nominal power drives a maximum flow of $3000 \mathrm{~m}^{3} / \mathrm{h}$ through the boundary layer wind tunnel exit section.

In the present study each jet unit of $15 \mathrm{~mm}$ of inner diameter is mounted vertically in the top of the test section 20.1D above the ground plane with the axis contained in the vertical plane of symmetry parallel to the crossflow as showed schematically in Fig. 6.

The origin of the horizontal, $\mathrm{X}$ and vertical, $\mathrm{Y}$, coordinates is taken at the midpoint between the centers of the jets exit. The $\mathrm{X}$ coordinate is positive in the direction of the wind tunnel exit and $\mathrm{Y}$ is positive upwards. The present results were obtained at the vertical plane of symmetry for jet mean velocity of $\mathrm{V}_{\mathrm{j}}=36 \mathrm{msec}$, a jet Reynolds number of $\mathrm{Re}_{\mathrm{j}}=4.3 \times 10^{4}$ and mean crossflow velocities of $U_{0}=0.8 \mathrm{msec}$, corresponding to a velocity ratio, $V_{R}=V_{j} / U_{0}=43.8$.

Visualization technique: Flow visualization was performed using digital direct photography to guide the choice of the measurement locations and to provide a qualitative picture of the flow.
The longitudinal plane of symmetry was illuminated with a sheet of light. This sheet was used to illuminate any cross section that has been seeded with seeding particles. The laser light reflected from the seeding particles but dark images was observed where there was an absence of seeding particles. The photos were taken perpendicular to the vertical plane of symmetry.

Computational method: This study presents a numerical analysis based on the experimental data presented by Vieira et al. (2013). The objective of the numerical simulation is to compare the numerical predictions with the experimental data in order to quantify the performance of the computational model and to extend the analysis of the flow.

Mathematical model: The mathematical model used in the numerical simulation is based on the solution of the continuity and momentum equations. A Reynolds-Averaged Navier Stokes (RANS) formulation was adopted with the "k- $\varepsilon$ " Turbulence Model to represent the turbulent stresses. The governing equations are written in a similar form:

$$
\frac{\partial}{\partial \mathrm{x}}(\rho \mathrm{u} \phi)+\frac{1}{\mathrm{r}} \frac{\partial}{\partial \mathrm{r}}(\mathrm{r} \rho \mathrm{v} \phi)=\frac{\partial}{\partial \mathrm{x}}\left(\Gamma_{\phi} \frac{\partial \phi}{\partial \mathrm{x}}\right)+\frac{1}{\mathrm{r}} \frac{\partial}{\partial \mathrm{r}}\left(\mathrm{r} \Gamma_{\phi} \frac{\partial \phi}{\partial \mathrm{r}}\right)+\mathrm{S}_{\phi}
$$

where, the property $\phi$ represents the velocity, turbulent kinetic energy or dissipation while $S_{\phi}$ and $\Gamma_{\phi}$ assume different values related with $\phi$ as described in Table 1 . The turbulent diffusion terms are approximated by two equations from " $k-\varepsilon$ " Turbulent Model where the Reynolds tension is related with shear tension: 
Table 1: Differential equations coefficients

\begin{tabular}{lll}
\hline$\phi$ & $\Gamma_{\phi}$ & $\mathrm{S}_{\phi}$ \\
\hline 1 & 0 & 0 \\
$\mathrm{U}$ & $\mu_{\mathrm{T}}$ & $-\frac{\partial \mathrm{p}}{\partial \mathrm{x}}+\frac{\partial}{\partial \mathrm{x}}\left(\mu_{\mathrm{T}} \frac{\partial \mathrm{U}}{\partial \mathrm{x}}\right)+\frac{1}{\mathrm{r}} \frac{\partial}{\partial \mathrm{r}}\left(\mathrm{r} \mu_{\mathrm{T}} \frac{\partial \mathrm{V}}{\partial \mathrm{x}}\right)$ \\
$\mathrm{V}$ & $\mu_{\mathrm{T}}$ & $-\frac{\partial \mathrm{p}}{\partial \mathrm{r}}+\frac{\partial}{\partial \mathrm{x}}\left(\mu_{\mathrm{T}} \frac{\partial \mathrm{U}}{\partial \mathrm{r}}\right)+\frac{1}{\mathrm{r}} \frac{\partial}{\partial \mathrm{r}}\left(\mathrm{r} \mu_{\mathrm{T}} \frac{\partial \mathrm{V}}{\partial \mathrm{r}}\right)-2 \mu_{\mathrm{T}} \frac{\mathrm{V}}{\mathrm{r}^{2}}$ \\
$\mathrm{k}$ & $\mu_{\mathrm{T}} / \sigma_{\mathrm{k}}$ & $\Phi-\rho \varepsilon$ \\
$\epsilon$ & $\mu_{\mathrm{T}} / \sigma_{\mathrm{k}}$ & $\mathrm{C}_{\varepsilon 1} \Phi \frac{\varepsilon}{\mathrm{k}}-\mathrm{C}_{\varepsilon 2} \rho \frac{\varepsilon^{2}}{\mathrm{k}}$ \\
\hline
\end{tabular}

$\Gamma_{\phi}:$ Transport coefficient; $\varepsilon$ : Turbulent kinetic energy dissipation; $\mu_{\mathrm{T}}$ : Turbulent viscosity; $\rho$ : Density; $\sigma_{\mathrm{k}}, \sigma_{\varepsilon}$ : Turbulent prandt1/schmidt numbers; $\phi$ : Variable in general conservation equation; $\Phi$ : Turbulent kinetic energy production term; $\mathrm{j}$ : Jet-exit value; $\mathrm{o}$ : Crossflow value

Table 2: Turbulent Model constants

\begin{tabular}{lcccc}
\hline $\mathrm{C}_{\mu}$ & $\mathrm{C}_{1}$ & $\mathrm{C}_{2}$ & $\sigma_{\mathrm{k}}$ & $\sigma_{\mathrm{E}}$ \\
\hline 0.09 & 1.44 & 1.92 & 1.0 & 1.3 \\
\hline
\end{tabular}

$$
\rho \overline{u_{i}^{\prime} u_{j}^{\prime}}=-\mu_{T}\left(\frac{\partial U_{i}}{\partial x_{j}}+\frac{\partial U_{j}}{\partial x_{i j}}\right)+\frac{2}{3} \delta_{i j} \rho k
$$

where, $\mu_{\mathrm{T}}$ represent turbulent viscosity derivative from the Turbulent Model expressed by:

$$
\Phi=\mu_{\mathrm{T}}\left\{2\left[\left(\frac{\partial \mathrm{U}}{\partial \mathrm{x}}\right)^{2}+\left(\frac{\partial \mathrm{V}}{\partial \mathrm{r}}\right)^{2}+\left(\frac{\mathrm{V}}{\mathrm{r}}\right)^{2}\right]+\left[\frac{\partial \mathrm{U}}{\partial \mathrm{r}}+\frac{\partial \mathrm{V}}{\partial \mathrm{x}}\right]^{2}\right\}
$$

Where:

$\mathrm{k}=$ Turbulent kinetic energy

$\mathrm{r}=$ Radius of cylindrical coordinates

$\mathrm{S}_{\phi}=$ Source term

$\mathrm{U}=$ Horizontal velocity, $\overline{\mathrm{U}}+\mathrm{u}^{\prime}\left(\mathrm{U}_{\text {mean }}+\mathrm{u}^{\prime}\right)$

$\mathrm{V}=$ Vertical velocity, $\overline{\mathrm{V}}+\mathrm{v}^{\prime}\left(\mathrm{V}_{\text {mean }}+\mathrm{v}^{\prime}\right)$

$\mathrm{W}=$ Transverse $\overline{\mathrm{W}}+\mathrm{W}^{\prime}\left(\mathrm{W}_{\text {mean }}+\mathrm{W}^{\prime}\right)$

$\mathrm{X}=$ Horizontal coordinate

$\mathrm{Y}=$ Vertical coordinate

$Z$ = Transverse coordinate

The computational domain (Fig. 7) corresponds to the experimental conditions and has $201 \mathrm{~mm}$ of transversal length, remaining constant the following measurements as followed: $1080 \mathrm{~mm}$ of longitudinal length, $402 \mathrm{~mm}$ of height where the inner diameter of the jets $\mathrm{D}$ is $15 \mathrm{~mm}$, the spacing between jets are $\mathrm{S}=6 \mathrm{D}$ and the height of impact, $\mathrm{H}$ is $20.1 \mathrm{D}$. The Turbulent Model constants allow good results for several types of flows and are summarized in Table 2. To reduce the computational costs and to reduce the time simulation of the flow, we only simulation half of the test section.

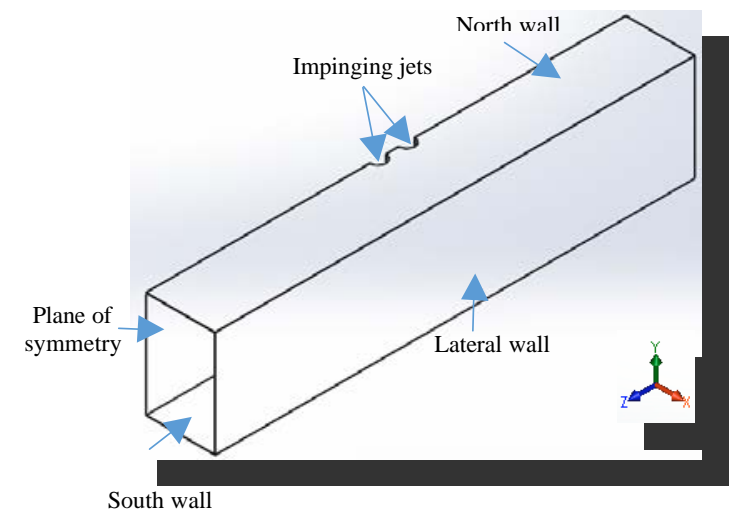

Fig. 7: Computational domain used in the numerical simulation

\section{RESULTS AND DISCUSSION}

The results presented and discussed in this section are to the flow visualization for the velocity ratio $V_{R}$ of 43.8 .

Visualization results: For the flow studied, the result has shown a pattern similar to that of a single impinging jet. Figure 8 identifies the flow development along the vertical plane of symmetry, i.e., $Z=0$. Each jet has an initial potential-core jet region where the flow characteristics are identical to those of a free jet and near the horizontal plate the impingement region, characterized by considerable deflection of the first jet. Analysing Fig. 8, it is clear that for this velocity ratio the deflection of the first jet is small. In the case of the rear jet deflection, we can verify that it is quite small compared with the first jet deflection because the first jet protects the rear jetof the influence of the crossflow and there was not impact of the rear jet on the ground for the lower velocity ratio. However, it is notorious a drop (Fig. 8c) in the rear jet potential core region that it has not been reported in literature. The horseshoe vortex resulting from the interaction of the upstream wall jet with the crossflow could not be clearly identified for this velocity ratio. The nature of each ground vortex is similar to the horseshoe structure known to be generated by the deflection of aboundary layer by a solid obstacle but is different from the vortex pair known to exist in a "bent-over" jet in a crossflow far from the ground. Figure 8e shows that the crossflow is deflected sideways by the penetration of the jet and may cause a recirculation region just downstream of the discharge, away from the ground plate but cannot be clearly identified. Also, it is possible see a little ground vortex and the wall jet corresponding to the upstream impinging jet which is almost parallel the ground plate and 


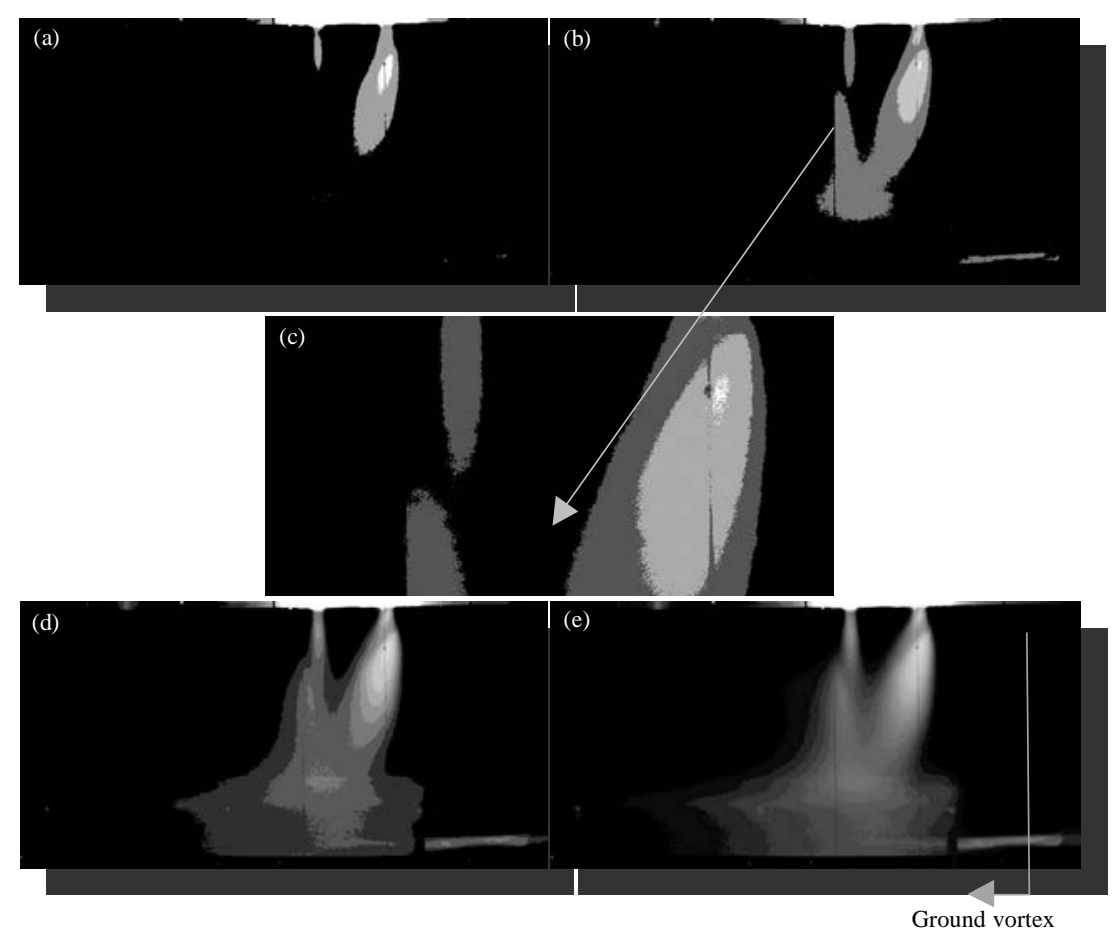

Fig. 8: a-e) Visualization of the twin jet flow in the vertical plane of symmetry in different phases of the flow development for $\mathrm{Re}_{\mathrm{j}}=4.3 \times 10^{4}, \mathrm{H} / \mathrm{D}=20.1$ and $\mathrm{S} / \mathrm{D}=6$

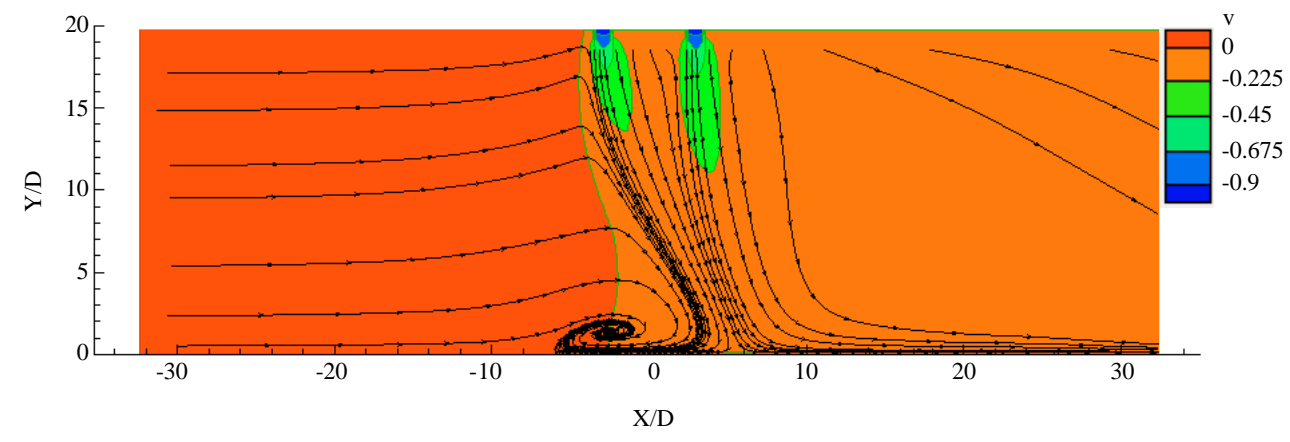

Fig. 9: Calculated stream traces and mean vertical velocity component distribution along the vertical plane of symmetry (i.e., $\mathrm{Z}=0$ ). $\mathrm{V}_{\mathrm{j}} / \mathrm{U}_{0}=22.5, \mathrm{Re}_{\mathrm{j}}=43,000, \mathrm{H} / \mathrm{D}=20.1$ and $\mathrm{L} / \mathrm{D}=6$

exhibit a behaviour similar to that of a radial wall jet where the upstream effects of interaction due to impingement are no longer important. The upstream wall jet interacts with the crossflow and forms a horseshoe vortex close to the ground plate which wraps mostly around the first impinging jet. As a result, two stream wise counter-rotating vortices develop side-to-side and decay further downstream of each impinging zone forming a ground vortex. If the jets were positioned side by side in front of the crossflow two ground vortexes would appear as well as a fountain flow in the vertical plane of symmetry due to the collision of the two individual radial wall jets
(Barata, 1996a, b; Saripalli, 1987). No evidence of a ground vortex corresponding to the downstream impinging jet could be confirmed which is an indication that the upstream impinging jet and its ground vortex are blocking the crossflow and provoking an alteration to the flow pattern. Also in the present study no fountain flow was detected.

Numerical results: This study presents a numerical study to extend the analysis of the flow to regions and flow conditions for which no measurements have been obtained. The numerical method is based on the solution 


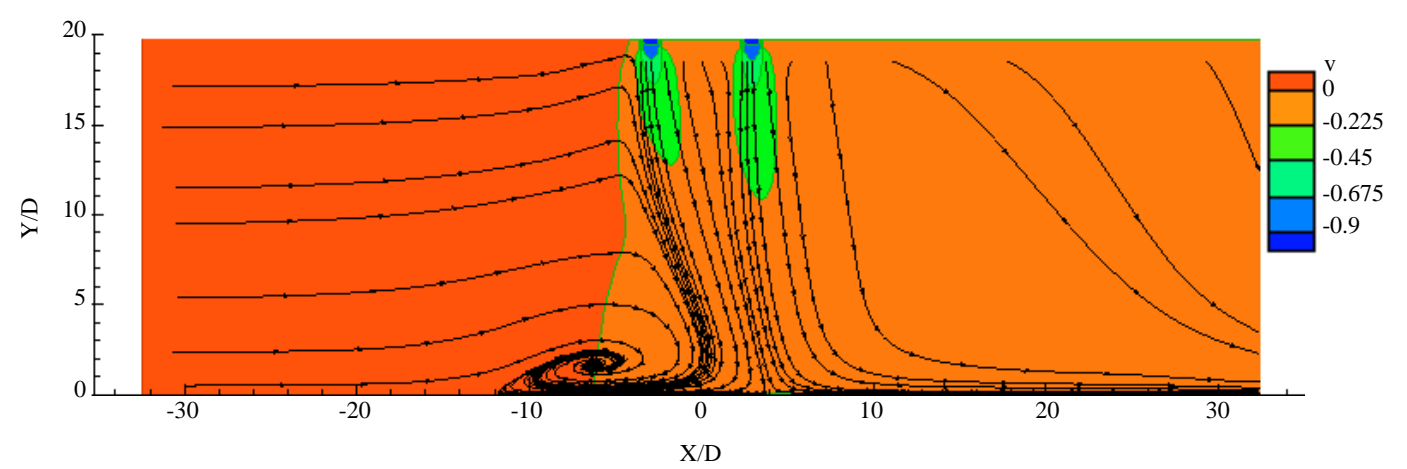

Fig. 10: Calculated stream traces and mean vertical velocity component distribution along the vertical plane of symmetry (i.e., $\mathrm{Z}=0$ ). $\mathrm{V}_{\mathrm{j}} / \mathrm{U}_{0}=33.7, \mathrm{Re}_{\mathrm{j}}=43,000, \mathrm{H} / \mathrm{D}=20.1$ and $\mathrm{L} / \mathrm{D}=6$

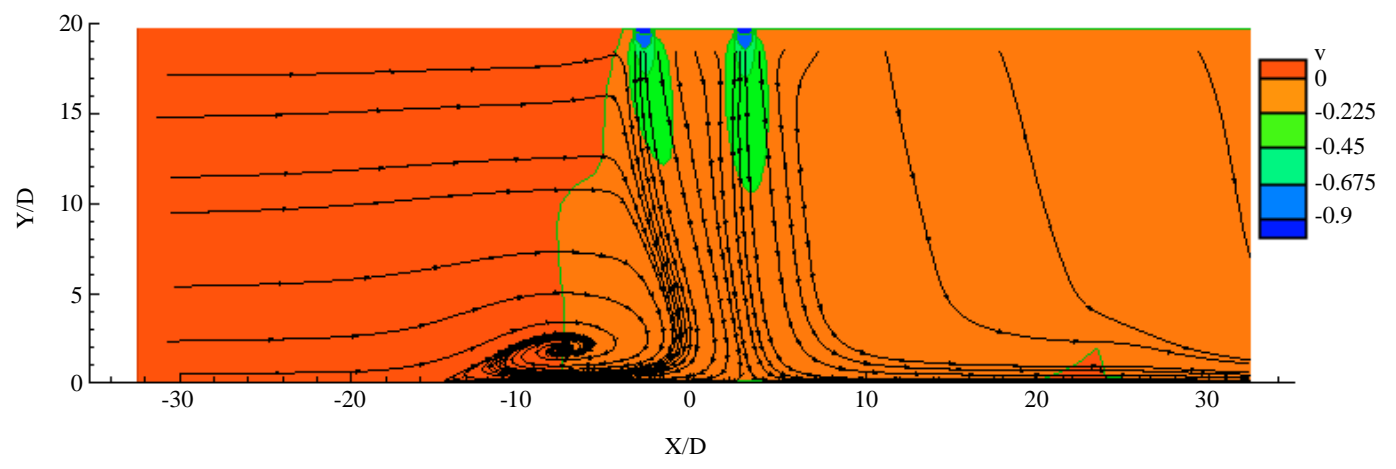

Fig. 11: Calculated stream traces and mean vertical velocity component distribution along the vertical plane of symmetry (i.e., $\mathrm{Z}=0$ ). $\mathrm{V}_{\mathrm{j}} / \mathrm{U}_{0}=43.8, \mathrm{Re}_{\mathrm{j}}=43,000, \mathrm{H} / \mathrm{D}=20.1$ and $\mathrm{L} / \mathrm{D}=6$

of the conservation laws for mass and momentum which is a modified version of the method described else where (Barata et al., 1989).

Figure 9-11 show the mean vertical velocity component $\left(\mathrm{V}_{\text {mean }}\right)$ distribution along the vertical plane of symmetry (i.e., $Z=0$ ) together with calculated stream traces. For all velocity ratios the collision of the upstream wall jet with the crossflow is clearly registered. Meanwhile, the ground vortex is also identified for all velocity ratios but its centre change with the velocity ratio applied because the increase of the velocity ratio corresponds to a decrease in crossflow velocity. Comparing this result with that obtained experimentally (Vieira et al., 2013, 2014) we can confirm that the deflection sensed by the first jet experimentally is greater than the jet deflection felt numerically thus leading for example for $V_{j} / U_{0}=33.7$, the centre of the ground vortex is located close to $\mathrm{X} / \mathrm{D}=-4$ in the experimental case, whereas for the numerical case it is located close to $\mathrm{X} / \mathrm{D}=-7$. As far as the downstream jet (rear jet) is concerned the predictions confirmed the experimental results (Vieira et al., 2013, 2014) and its complete deflection by the crossflow was calculated for all the velocity ratios considered with no impingement directly on the wall. Figure 12 shows in more detail the central zone between the two jets with white stream traces that begin near the top wall. The results show that even for the smallest velocity ratios of $\mathrm{V}_{\mathrm{j}} / \mathrm{U}_{0}=7.5$ and 15 the jets do not mix but remain together in two layers. As a consequence for $V_{j} / U_{0}=15$ the downstream jet does not reach directly the ground but it impinges on the wall jet resulting from the first jet which is moving downstream. For the two higher velocity ratios the ground vortex is always present but its size and location changes. It moves upstream with $\mathrm{V}_{\mathrm{j}} / \mathrm{U}_{0}$ because increasing the velocity ratio in volves decreasing the relative influence of the crossflow velocity. Barata (2013a, b) identified three different types of flow regimes but for a V/STOL aircraft operating in ground vicinity the regime with strong impingement on the ground and a ground vortex is the most relevant. However in transition from hover to horizontal flight the other regimes are also important.

One major issue in the present flow configuration is the possible deflection of the rear jet by the first jet 

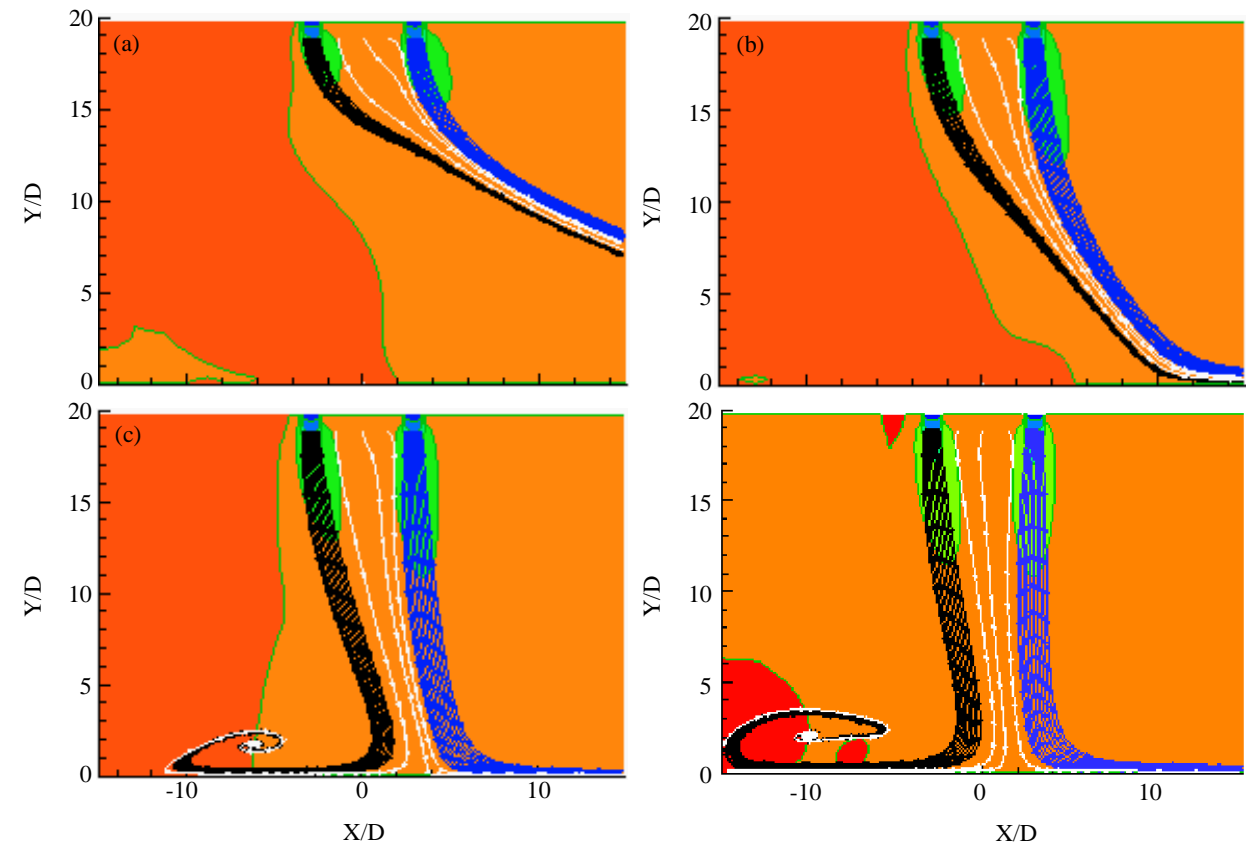

Fig. 12: Details of the calculated stream traces and mean vertical velocity component distribution along the vertical plane of symmetry (i.e., $\mathrm{Z}=0$ ). $\mathrm{Re}_{\mathrm{j}}=4.3 \times 10^{4}, \mathrm{H} / \mathrm{D}=20.1$ and $\mathrm{L} / \mathrm{D}=6$ : a) $\left.\mathrm{V}_{\mathrm{j}} / \mathrm{U}_{0}=7.5 ; \mathrm{b}\right) \mathrm{V}_{\mathrm{j}} / \mathrm{U}_{0}=15.0 ;$ c) $\mathrm{V}_{\mathrm{j}} / \mathrm{U}_{0}=33.7$ and d) $\mathrm{V}_{\mathrm{j}} / \mathrm{U}_{0}=60.0$

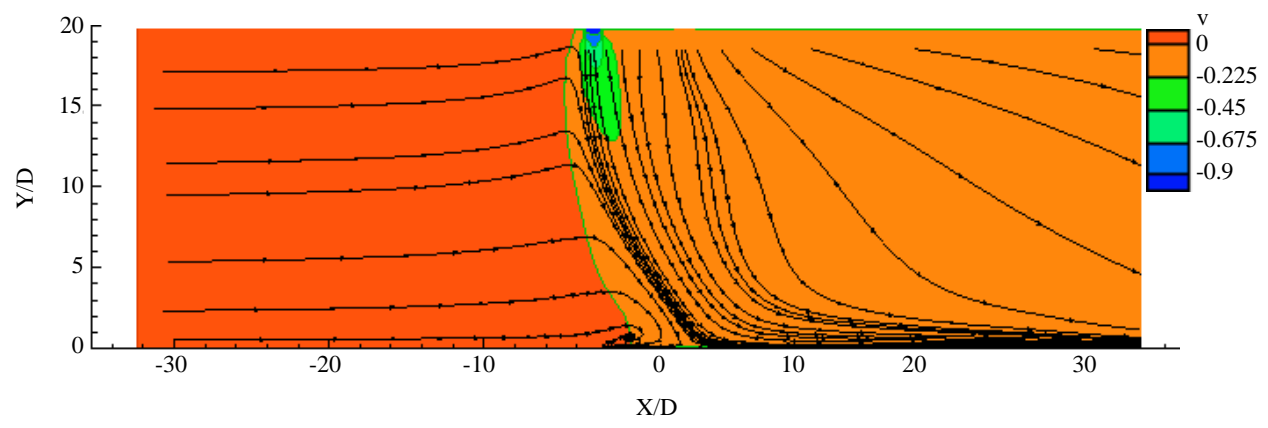

Fig. 13: Calculated stream traces and mean vertical velocity component distribution along the vertical plane of symmetry, $\mathrm{Z}=0 . \mathrm{Re}_{\mathrm{j}}=43,000, \mathrm{H} / \mathrm{D}=20.1, \mathrm{~L} / \mathrm{D}=6, \mathrm{~V}_{\mathrm{j} 2}=5.4 \mathrm{~m} \mathrm{sec}^{-1}$

without occurring impact on the ground. Another possibility (not yet demonstrated) is the impact of the second jet with the first deflected jet or its downstream wall (depending on the velocity ratio).

Any of the figure presented above it is sufficiently conclusive about these hypothesis nor about the blending of the two structures further downstream. In order to better understand this type of flow and in particular that happened with the rear jet and downstream of the impinging jets, a meticulous numerical study simulation was performed for $\mathrm{V}_{j} / \mathrm{U}_{0}=22.5$ by varying the velocity of the second jet, $V_{j 2}$ from $5.4 \mathrm{~m} \mathrm{sec}^{-1}$ up to $36 \mathrm{~m} \mathrm{sec}^{-1}$. The velocity of the first jet was kept constant to preserve the location of the centre of the ground vortex.
Despite being varied the second jet velocity from 5.4, up to $36 \mathrm{~m} \mathrm{sec}^{-1}$, it was only possible to view significant changes on the flow to $V_{\mathrm{j} 2} \leq 13.5 \mathrm{~m} \mathrm{sec}^{-1}$. Figure 13 and 14 show the results for $\mathrm{V}_{\mathrm{j} 2}=5.4$ and $13.5 \mathrm{~m} \mathrm{sec}^{-1}$, respectively.

Both figures show that lowering the velocity of the second jet in relation to the first jet, the wall jet of the second jet is always above the wall jet formed by the interaction of the first jet with the crossflow, heading the flow to downstream. The location of the ground vortex is practically not affected by this change in the second jet velocity, confirming that the second jet does not contribute to the occurrence of this structure.

In order to investigate the possible existence of a kidney shape of the crossection of the jets and their 


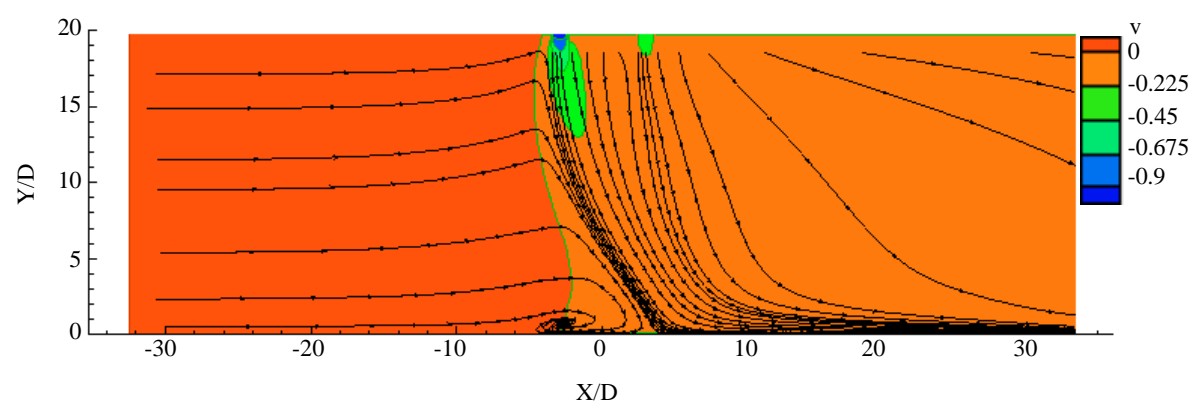

Fig. 14: Calculated stream traces and mean vertical velocity component distribution along the vertical plane of symmetry, $\mathrm{Z}=0 . \mathrm{Re}_{\mathrm{j}}=43,000, \mathrm{H} / \mathrm{D}=20.1, \mathrm{~L} / \mathrm{D}=6, \mathrm{~V}_{\mathrm{j} 2}=13.5 \mathrm{~m} \mathrm{sec}^{-1}$
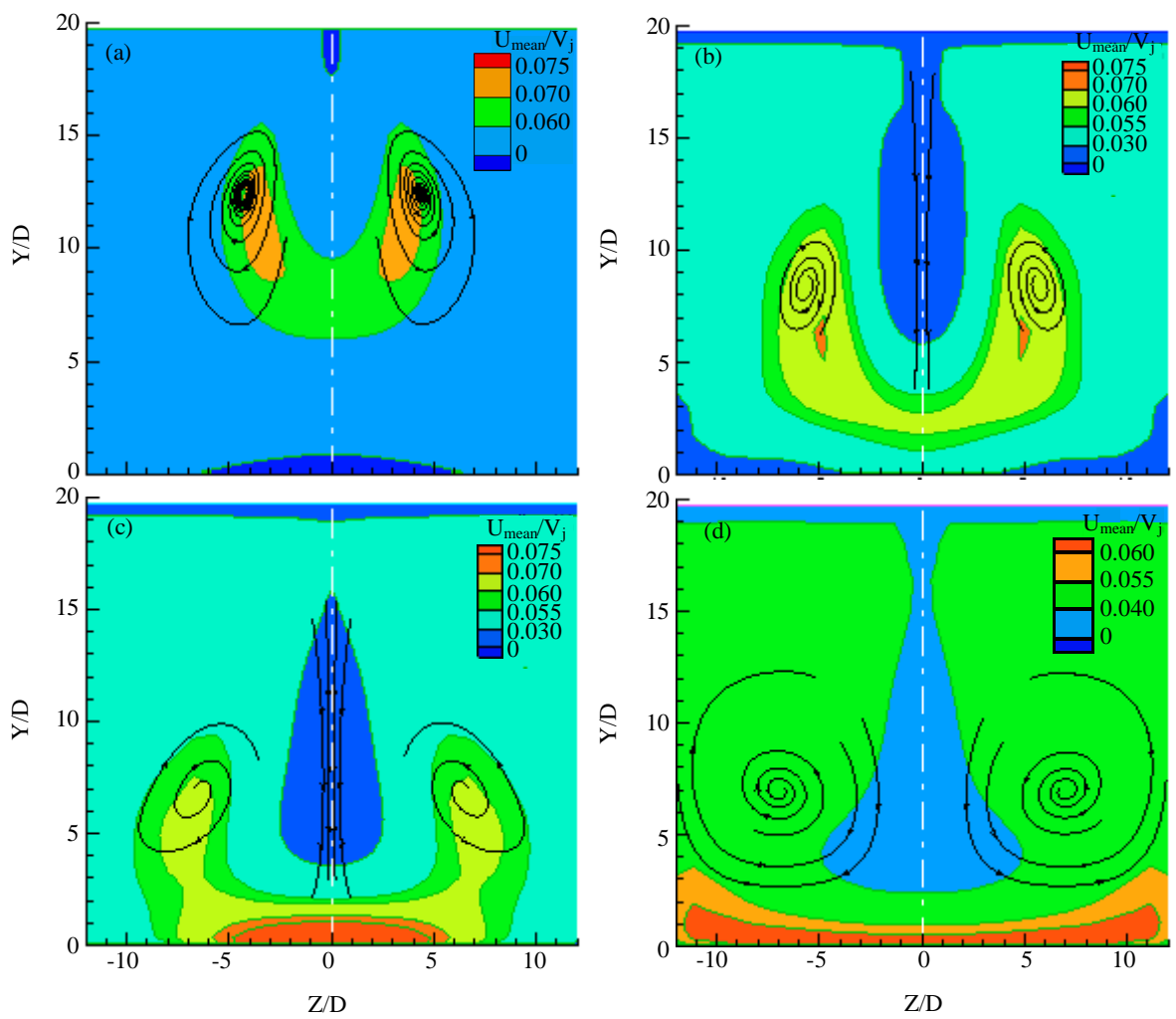

Fig. 15: Mean velocity component $\left(\mathrm{U}_{\text {mean }} / V_{\mathrm{j} 1}\right)$ in a vertical plane perpendicular to crossflow $\left(\operatorname{Re}_{j 1}=43,000\right.$, $\left.\mathrm{V}_{\mathrm{j} 2}=5.4 \mathrm{~m} \mathrm{sec}^{-1}, \mathrm{Re}_{\mathrm{j} 2}=5,339, \mathrm{H} / \mathrm{D}=20.1, \mathrm{~L} / \mathrm{D}=6\right):$ a) $\mathrm{X} / \mathrm{D}=0.0$; b) $\mathrm{X} / \mathrm{D}=4.9$; $) \mathrm{X} / \mathrm{D}=9.8$ and d) $\mathrm{X} / \mathrm{D}=19.6$

mutual interactions, isolines of the velocity component $\mathrm{U}_{\text {mean }}$ were obtained in vertical planes perpendicular to the crossflow for $\mathrm{X} / \mathrm{D}=0.0,4.9,9.8$ and 19.6. Figure 15 and 16 show the results for $V_{j 2}=5.4$ and $V_{j 2}=13.5 \mathrm{~m} \mathrm{sec}^{-1}$, respectively. For $\mathrm{X} / \mathrm{D}=0$, i.e. in the middle between the axis of the jets exit, the kidney shape can be clearly identified for the first jet (the second jet it is not yet visible because its exit is further downstream).

For $\mathrm{X} / \mathrm{D}=4.9$, the second jet can be identified by the dark blue area corresponding to velocities larger than $0.03 \mathrm{~V}_{\mathrm{j} 1}$ that reveals the slight deflection of the second jet inside the wake of the first impinging jet. The kidney shape of the cross section of the first jet is still present but it is widening rapidly due to the additional influence of the second imping jet. It should be pointed out that the impinging jet and first jet do not mix and are separated by lower values of the velocity component in the crossflow direction (light blue). Additionally, no stream traces from the second jet are captured by the deflected upstream jet, revealing that a complete mixing between the two jets has not yet occurred. However, from this location downstream (in the crossflow direction) the second impinging jet will 

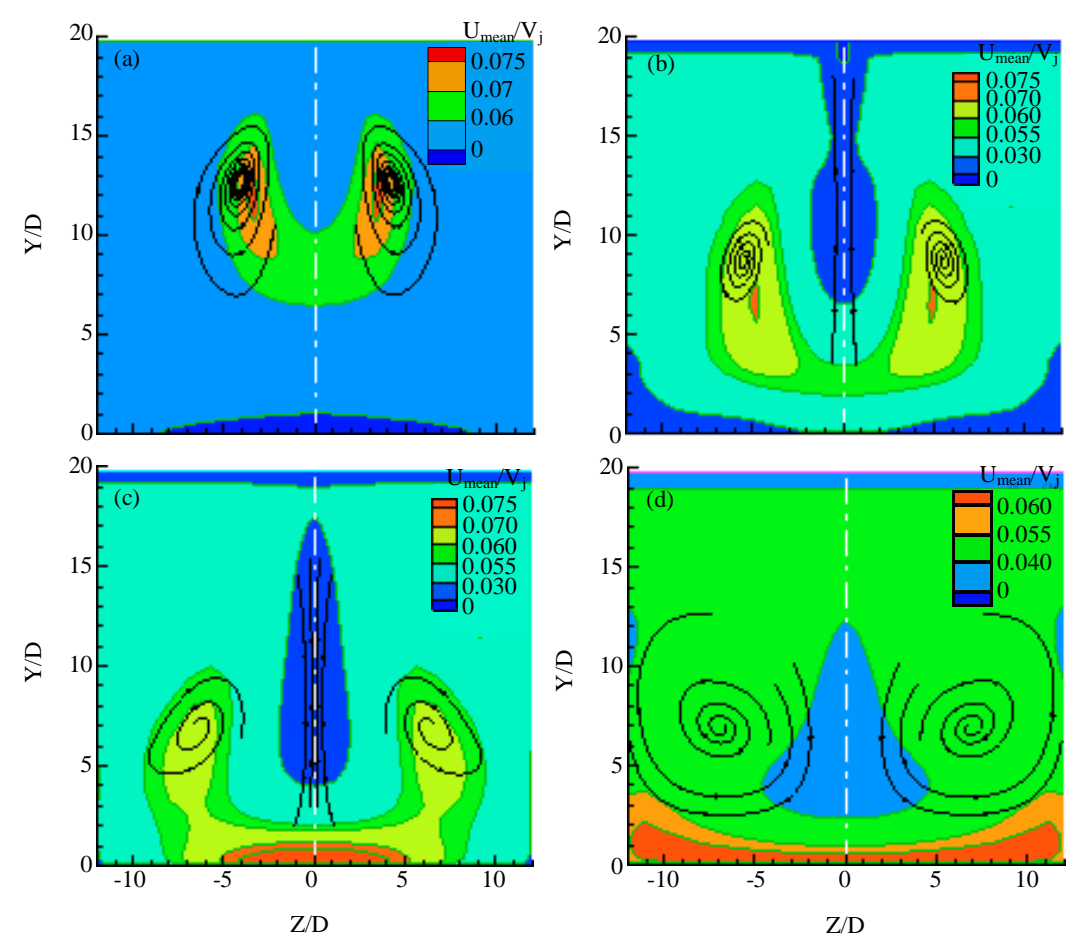

Fig. 16: Mean velocity component $\left(\mathrm{U}_{\text {mean }} / \mathrm{V}_{\mathrm{j} 1}\right)$ in a vertical plane perpendicular to crossflow $\left(\operatorname{Re}_{\mathrm{j} 1}=43,000\right.$, $\mathrm{V}_{\mathrm{j} 2}=13.5 \mathrm{~m} \mathrm{sec}^{-1}, \mathrm{Re}_{2}=16,125, \mathrm{H} / \mathrm{D}=20.1, \mathrm{~L} / \mathrm{D}=6$ ): a) $\left.\left.\mathrm{X} / \mathrm{D}=0.0 ; \mathrm{b}\right) \mathrm{X} / \mathrm{D}=4.9 ; \mathrm{c}\right) \mathrm{X} / \mathrm{D}=9.8$ and d) $\mathrm{X} / \mathrm{D}=19.6$

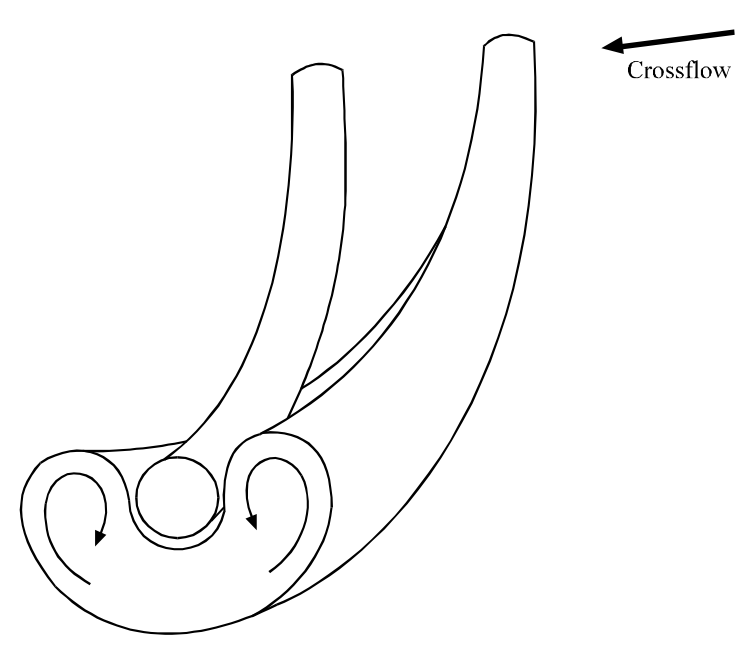

Fig. 17: Illustration of the kidney shape of the cross section of the first jet before reaching the ground and the second (downstream) jet interaction

suffer two opposing effects: first the fact that is developing in the wake of the first impinging jet will contribute to its less deflection and secondly since it is impinging in a flow moving in the crossflow direction with a higher velocity it will be forced to deflect. This novel flow configuration that occurs before the first jet reaching the ground is schematically shown in Fig. 17. So, the final behaviour of the second jet can be described as the flow of a jet through a zero or small upstream crossflow (corresponding to the wake of the first jet) impinging on a horizontal jet with a kidney cross section flowing in the crossflow direction with a higher velocity. In the next downstream location, at X/D $=9.8$, the red area identifies a region with relatively large positive values of the velocity component $U_{\text {mean }}$ (in the crossflow direction) which is an indication that the first jet touched the ground. The rear jet impacts on this wall jet (resulting from the first jet) and is quickly deflected but does not reach the ground. For $\mathrm{X} / \mathrm{D}=19.6$, the entire cross section is occupied by two vortical structures rotating in opposite directions, revealing that the two parallel jets flowing in the crossflow direction finally merge.

\section{CONCLUSION}

The flow field created by ground vortex generated by twin impinging jets in tadem through a crossflow is studied in detail. Flow visualization and numerical simulation are presented for two turbulent circular jets impinging on a flat surface perpendicular to the geometrical nozzle axis, through a low velocity crossflow. The numerical simulation were performed 
for Reynolds based on the jet exit conditions of $43,000-105,000$, a jet-to-crossflow velocity ratio between 15 and 60 being the focus of the study the velocity ratio used in experimental research (Vieira et al., 2013, 2014) for an impinging height of 20.1 diameters (the same used in experimental work) and with an interjet spacing of $S=6 \mathrm{D}$ (the same used in experimental work).

The results show a large penetration of the first (upstream) jet which is deflected by the crossflow and impinges on the ground giving rise to a ground vortex due to the collision of the radial wall and the crossflow that wraps around the impinging point like a scarf. The first jet deflection and the location of the ground vortex depend on the velocity ratioused. For higher velocity ratios the deflection of the first jet is smaller and closer to the first jet is located the centre of the ground vortex. The rear jet it is not so affected by the crossflow in terms of deflection for all velocity ratios because it is protected by the upstream jet but due to the downstream wall jet that flows radially from the impinging point the first jet does not reach the ground. Also due to the confinement and the ground vortex, the crossflow is blocked and accelerates in the upper part and also contributes to an enhanced mixing of each secondary flow. As consequence, no upstream wall jet or ground vortex resulting from the second (downstream) jet was detected. The effect of the rear jet impinging on the downstream wall jet resulting from the first jet had not been reported so far and requires further investigation.

In case of the numerical analysis beyond the rig experimental limits there sults show that for the two lowest velocity ratios, the jets do not mix remaining in two layers together and therefore, it is not detected the presence of ground vortex. For $\mathrm{V}_{\mathrm{j}} / \mathrm{U}_{0}=33.7$ and 60 the ground vortex is completely present and it moves with the increase of $\mathrm{V}_{\mathrm{j}} / \mathrm{U}_{0}$.

As far as the downstream jet (rear jet) is concerned the predictions confirmed the experimental results obtain by Vieira et al. (2013) and its complete deflection by the crossflow was calculated for all the velocity ratios considered with no impingement directly on the wall. By changing the rear jet velocity and extracting perpendicular planes to the jets flow was possible to prove that the second jet is deflected quickly, never reaching the ground thus proving the accuracy of the experimental results, leading us to conclude that impinging jet in tandem configuration, the downstream jet is entrained by the upstream jet and not by the crossflow itself. In the future, this issue should be further investigated in order to understand what the real consequence for the air craft when this type of phenomenon occurs.

\section{ACKNOWLEDGEMENTS}

The assistance of Ms. Ines Carvalho with some illustrations of the flow field is gratefully acknowledged. The present research was done in the scope of the activities of LAETA-Associated Laboratory of Energy, Transports and Aeronautics and funded by FCT-Fundacao para a Ciencia e Tecnologia through contract PTDC/EME-MFE/102190/2008 and grant $\mathrm{SFRH} / \mathrm{BD} / 86434 / 2012$.

\section{REFERENCES}

Abdel-Fattah, A., 2007. Numerical and experimental study of turbulent impinging twin-jet flow. Exp. Therm. Fluid Sci., 31: 1061-1072.

Adler, D. and A. Baron, 1979. Prediction of a three-dimensional circular turbulent jet in crossflow. AIAA J., 17: 168-174.

Aldabbagh, L.B.Y. and A.A. Mohamad, 2006. Effect of jet-to-plate spacing in laminar array jets impinging. Heat Mass Trans., 43: 265-273.

Aldabbagh, L.B.Y. and I. Sezai, 2002a. Numerical simulation of three-dimensional laminar multiple impinging square jets. Int. J. Heat Fluid Flow, 23: 509-518.

Aldabbagh, L.B.Y. and I. Sezai, 2002b. Numerical simulation of three-dimensional laminar, square twinjet impingement on a flat plate, flow structure and heat transfer. Numeric. Heat Trans. Part A: Applic.: Int. J. Comput. Methodol., 41: 835-850.

Barata, J.M.M., 1989. Numerical and experimental study of jets impinging on flat surfaces through a crossflow. Ph.D. Thesis, Technical University of Lisbon, Lisbon, Portugal.

Barata, J.M.M., 1996a. Fountain flows produced by multiple impinging jets in a crossflow. AIAA J., 34: 2523-2530.

Barata, J.M.M., 1996b. Ground vortex formation with twin impinging jets. Proceedings of the International Powered Lift Conference, November 18-20, 1996, Jupiter, Florida.

Barata, J.M.M., 2013a. Multiple jet/wall/cross-wind interaction relevant to VSTOL ground effects. Proceedings of the International Powered Lift Conference, August 12-14, 2013, Los Angeles, California.

Barata, J.M.M., 2013b. Twin impinging jets aligned with a crossflow. Proceedings of the 49th AIAA/ASME/ $\mathrm{SAE} / \mathrm{ASEE}$ Joint Propulsion Conference and Exhibit, July 15-17, 2013, San Jose, California.

Barata, J.M.M., D.F.G. Durao and J.J. McGuirk, 1989. Numerical study of single impinging jets through a crossflow. J. Aircraft, 26: 1002-1008. 
Barata, J.M.M., D.F.G. Durao and J.J. McGuirk, 1991. Impingement of single and twin turbulent jets through a crossflow. AIAA J., 29: 595-602.

Barata, J.M.M., D.F.G. Durao, M.V. Heitor and J.J. McGuirk, 1993. On the analysis of an impinging jet on ground effects. Exp. Fluids, 15: 117-129.

Behrouzi, P. and J.J. McGuirk, 2000. Experimental data for CFD validation of the intake ingestion process in STOVL aircraft. Flow Turbulence Combustion, 64: $265-277$.

Caliskan, S. and S. Baskaya, 2012. Velocity field and turbulence effects on heat transfer characteristics from surfaces with V-Shaped ribs. Int. J. Heat Mass Trans., 55: 6260-6277.

Crabb, D., D.F.G. Durao and J.H. Whitelaw, 1981. A round jet normal to a crossflow. J. Fluids Eng., 113: 142-153.

Durst, F., A. Melling and J.H. Whitelaw, 1976. Principles and Practice of Laser-Doppler Anemometry. 2nd Edn., Academic Press, New York, USA., ISBN-13: 9780122252501 , Pages: 405.

Fan, J.Y., D.Z. Wang and Y. Zhang, 2006. Large-scale vortical structures produced by an impinging density jet in shallow crossflow. Applied Math. Mech., 27: 363-369.

Gilbert, B.L., 1983. Detailed turbulence measurements in a two opposing plane turbulent wall jets. Proceedings of the AIAA 16th Fluid and Plasma Dynamics Conference, July 12-14, 1983, Danvers, MA.

Jones, W.P. and J.J. McGuirk, 1980. Computation of a Round Turbulent Jet Discharging into a Confined Crossflow. In: Turbulent Shear Flows 2: Selected Papers from the Second International Symposium on Turbulent Shear Flows, Imperial College London, July 2-4, 1979, Bradbury, L.J.S. (Ed.). Springer Verlag, Heidelberg, German, ISBN-13: 9783540100676 , pp: 223-245.

Kamotani, Y. and I. Greber, 1974. Experiments on confined turbulent jets in cross flow. Technical Report, NASA-CR-2392; NAS 1.26:2392, Washington, DC., USA., March 1974.

Kate, R.P., P.K. Das and S. Chakraborty, 2007. An experimental investigation on the interaction of hydraulic jumps formed by two normal impinging circular liquid jets. J. Fluid Mech., 590: 355-380.

Knowles, K. and D. Bray, 1991. The ground vortex formed by impinging jets in crossflow. Proceedings of the AIAA 29th Aerospace Sciences Metting, January 7-10, 1991, Reno, NV.

Knowles, K., 1996. Computational studies of impinging jets using $\mathrm{k}-\varepsilon$ Turbulence Models. Int. J. Numer. Meth. Fluids, 22: 799-810.
Kolar, V. and E. Savory, 2007. Dominant flow features of twin jets and plumes in crossflow. J. Wind Eng. Ind. Aerodynamics, 95: 1199-1215.

Kolar, V., H. Takao, T. Todoroki, E. Savory, S. Okamoto and N. Toy, 2003. Vorticity transport within twin jets in crossflow. Exp. Therm. Fluid Sci., 27: 563-571.

Li, G., Y. Zheng, G. Hu and Z. Zhang, 2014. Convective heat transfer enhancement of a rectangular flat plate by an impinging jet in cross flow. Chin. J. Chem. Eng., 22: 489-495

Miao, J.M., C.Y. Wu and P.H. Chen, 2009. Numerical investigation of confined multiple-jet impingement cooling over a flat plate at different crossflow orientations. Numer. Heat Transfer Part A: Applic., 55: 1019-1050.

Nishino, K., M. Samada, K. Kasuya and K. Torii, 1996. Turbulence statistics in the stagnation region of an axisymmetric impinging jet flow. Int. J. Heat Fluid, 17: 193-201.

Patankar, S.V., D.K. Basu and S.A. Alpay, 1979. Prediction of the three-dimensional velocity field of a deflected turbulent jet. J. Fluids Eng., 99: 758-762.

Saddington, A.J., P.M. Cabrita and K. Knowles, 2005. Large-scale instabilities in a STOVL upwash fountain. Proceedings of the 6 th International Symposium on Engineering Turbulence Moddelling and Measurements, May 23-25, 2005, Cagliari, Italy.

Saripalli, K.R., 1983. Visualization of multijet impingement flow. AIAA J., 21: 483-484.

Saripalli, K.R., 1987. Laser Doppler Velocimeter Measurements in 3-D Impinging Twin-Jet Fountain Flows. In: Turbulent Shear Flows 5: Selected Papers from the Fifth International Symposium on Turbulent Shear Flows, Cornell University, Ithaca, New York, USA, August 7-9, 1985, Durst, F., B.E. Launder, J.L. Lumley, F.W. Schmidt and J.H. Whitelaw (Eds.). Springer-Verlag, Berlin, Germany, ISBN-13: 978-3-64271435-1, pp: 147-168.

Vieira, D.F.C., A.R.R. Silva, P. Carvalho, F. Neves and J.M.M. Barata, 2013. Numerical and experimental study of two impinging jets in a row through a crossflow. Proceedings of the AIAA 51th Aerospace Science Meeting Including the New Horizons Forum and Aerospace Exposition, January 7-10, 2013, Texas.

Vieira, D.F.C., D.F.G. Durao, F.M.S.P. Neves, A.R.R. Silva and J.M.M. Barata, 2014. Laser doppler measurements of twin impinging jets in tadenm through a crossflow. Proceedings of the 17 th International Symposium on Applications of Laser Techniques to Fluid Mechanics Lisbon, July 7-10, 2014, Portugal. 
Wae-Hayee, M., P. Tekasakul, S. Eiamsa-Ard and C. Nuntadusit, 2014. Effect of cross-flow velocity on flow and heat transfer characteristics of impinging jet with low jet-to-plate distance. J. Mech. Sci. Technol., 28: 2909-2917.

Worth, N.A. and Z. Yang, 2006. Simulation of an impinging jet in a crossflow using a Reynolds stress transport model. Int. J. Numer. Meth., 52: 199-211.
Yang, Y.T. and C.H. Shyu, 1998. Numerical study of multiple impinging slot jets with an inclined confinement surface. Numer. Heat Transfer Part A: Applic., 33: 23-27.

Yang, Z., 2014. Assessment of unsteady-RANS approach against steady-RANS approach for predicting twin impinging jets in a cross-flow. Congent Eng., Vol. 1. $10.1080 / 23311916.2014 .936995$. 